\title{
Association of Low Zinc Finger Antiviral Protein Expression with Progression and Poor Survival of Patients with Hepatocellular Carcinoma
}

\author{
Ying Liu ${ }^{a, b}$ Cheng Huc Wen-Bo Zhu ${ }^{d}$ Wen-Xiong Xua,b Zhan-Yi Lia,b \\ Yuan Lin ${ }^{d}$ Jing Cai ${ }^{d}$ Jian-kai Liang ${ }^{d}$ Xiang Zhua,b Zhi-Liang Gao ${ }^{a, b}$ \\ Guang-mei Yan ${ }^{d, e} \quad$ Liang Penga,b
}

\begin{abstract}
aDepartment of Infectious Diseases, Third Affiliated Hospital of Sun Yat-sen University, Guangzhou, ${ }^{b}$ Guangdong Key Laboratory of Liver Disease Research, the Third Affiliated Hospital of Sun Yatsen University, Guangzhou 'Department of Urology Diseases, Third Affiliated Hospital of Sun Yatsen University, Guangzhou, 'Departments of Pharmacology, Sun Yat-sen University, Guangzhou, eCollaborative Innovation Center for Cancer Medicine, Guangzhou, China
\end{abstract}

\section{Key Words}

Zinc antiviral protein $\bullet$ ZAP $\bullet$ Hepatocellular carcinoma $\bullet$ Prognosis $\bullet$ Biomarker

\begin{abstract}
Background/Aims: Zinc finger antiviral protein (ZAP) has been reported to be expressed in hepatocellular carcinoma (HCC), and ZAP expression is associated with apoptotic signaling in cancer cells. This study aimed at investigating the expression of ZAP in HCC cells and its significance in clinical pathology. Methods: Real-time quantitative PCR and western blot assays were employed to detect ZAP RNA and protein expression in normal human hepatocytes, HCC cells, and five primary HCC cell lines. Immunohistochemistry was performed to detect ZAP expression in 147 paraffin-embedded HCC tissues and adjacent normal tissues. The clinical significance of ZAP expression was analyzed in tissue samples from patients with or without infection by hepatitis B virus (HBV). Results: ZAP expression in HCC cells and human primary HCC cell lines was significantly lower than that of normal human hepatocytes. Among 147 HCC samples, ZAP expression was lower in HCC tissues than in adjacent normal tissues for 107 (77.0\%) samples. In patients with HCC and HBV infection, ZAP expression was related to pathological grade $(P<0.05)$; in HBV-negative patients with HCC, ZAP expression was associated with tumor size $(P<0.05)$ and clinical stage $(P<0.05)$. The overall survival time in patients with low ZAP expression was significantly shorter than survival times of those with high ZAP expression $(P<0.05)$, especially for patients with moderately to well-differentiated HCC (Grade 1-2) and HCC at stage T1 and T2 $(P<0.05)$. Cox multivariate analysis showed

L. Ying and $\mathrm{H}$. Cheng contributed equally to this work.

\begin{tabular}{ll}
\hline Liang Peng & Department of Infectious Diseases, Third Affiliated Hospital of Sun Yat-sen University, Guangzhou \\
and Guang-mei Yan & Guangdong 510630 (China) \\
& E-Mail pzp33@hotmail.com, ygm@mail.sysu.edu.cn
\end{tabular}
\end{abstract}


that ZAP expression was an independent predictor of survival of patients with $\mathrm{HCC}(P<0.01)$. Conclusion: Low ZAP expression is closely associated with disease progression and poor prognosis for patients with HCC.

(C) 2018 The Author(s)

Published by S. Karger AG, Basel

\section{Introduction}

Hepatocellular carcinoma (HCC) is the third most common malignancy worldwide and the second leading cause of cancer-related deaths [1]. The occurrence and development of HCC are associated with chronic hepatitis, alcoholism, and toxin-induced liver injury. Chronic liver injury may progress into hepatic cirrhosis and hyperplasic liver injury, leading to HCC [2]. Studies have shown that tumor genesis of HCC may be related to genetic and epigenetic factors that disrupt key cellular processes and cause malignant transformation of normal cells [3]. Molecular mechanisms at the transcriptional level may profoundly reflect the type of cancer a patient develops. Previous studies have revealed that HCC pathogenesis is related to activation of c-my and mutations of the tumor suppressor gene p53, cyclin D1 (CCND1), c-Met, and genes in the Ras/PI3 kinase signaling pathway [4-9]. However, the specific molecular genetic mechanisms underlying HCC pathogenesis is still poorly understood. Currently, there are no guidelines for the diagnosis and treatment of HCC that are based on genetic testing. Thus, it is important to investigate new targets in the pathogenesis, diagnosis, and treatment of HCC.

Zinc finger antiviral protein (ZAP), is an RNA-binding protein that regulates the stability and translation of specific mRNAs. This gene encodes a CCCH-type zinc finger protein that is thought to prevent infection by retroviruses. It exerts an antiviral effect by recognizing and binding to viral RNA, thereby recruiting the host RNA degradation system, which degrades or inhibits the translation of viral RNA [10]. Besides its antiviral effect, Todorova et al. [11] found that ZAP exerts an antitumor effect by binding to cellular RNA to prevent apoptosis induced by tumor necrosis factor-related apoptosis inducing ligand (TRAIL). In our previous study, we found that a decrease of ZAP expression in cancer tissue compared with pericarcinous tissue is common in liver, colon, and bladder cancer [12], suggesting that loss of ZAP may indeed be favorable for the progression of certain cancers. Bioinformatic analysis of the cBioPortal database (http://www.cbioprotal.org) also revealed that HCC exhibits low expression of ZAP mRNA. However, the clinical significance of ZAP in HCC has never been reported.

In this study, we examined expression of ZAP in HCC cell lines, primary HCC cells, and clinical HCC tissues; investigated the relationship between ZAP expression and clinical factors, including chronic infection with HBV, hepatic cirrhosis, levels of alpha-fetoprotein (AFP), tumor size, pathological grade, clinical stage, tumor-node-metastasis (TNM) stage, vascular invasion, and recurrence; and explored the relationship between ZAP deficiency and survival of patients with HCC.

\section{Materials and Methods}

Cell lines

HCC cell lines, including HEP-3B, HUH-7, HUH-6, Sun-387, Sun-449, SK-HEP-1, Sun-182, Li-7, PLC, HEP-G2, and Bel-7402, and the normal liver cell line L- 02 were maintained at $37^{\circ} \mathrm{C}$ with $5 \% \mathrm{CO}_{2}$ supplemented with $10 \%(v / v)$ fetal bovine serum and 1\% penicillin/streptomycin (Life Technologies, Shanghai, China). All cell lines were purchased from the American Type Culture Collection (Manassas, VA) and the Shanghai Institute of Cell Biology (Shanghai, China).

Primary normal hepatocyte cells were purchased from ScienCell Research Laboratories (Carlsbad, CA) and cultured according to the manufacturer's instructions.

Primary HCC cells were collected from the surgical specimens of 5 patients undergoing treatment at the Department of Hepatic Surgery, the Third Affiliated Hospital of Sun Yat-sen University (Guangzhou, 


\section{Cellular Physiology Cell Physiol Biochem 2018;49:1048-1059 \begin{tabular}{l|l|l} 
DOI: 10.1159/000493285 & (c) 2018 The Author(s). Published by S. Karger AG, Basel
\end{tabular} and Biochemistry Published online: 6 September, 2018 www.karger.com/cpb}

Ying et al.: Low ZAP Related with Progression and Poor Survival of HCC

China), in accordance with the rules and regulations of China concerning the ethical use of human subjects in biomedical research. Primary cancer cells were isolated from surgical tumor tissues using $0.1 \%$ trypsin and then cultured in medium (Dulbecco's modified Eagle's medium/Nutrient Mixture F-12, 1:1) supplemented with growth factor, $10 \%(\mathrm{v} / \mathrm{v})$ fetal bovine serum, and 1\% penicillin/streptomycin (Life Technologies).

\section{Quantitative reverse transcription-PCR}

Total RNA was extracted by using Trizol (Life Technologies) reagent in accordance with the manufacturer's instructions. The extracted RNA was pretreated with RNase-free DNase, and then reverse transcribed to cDNA with oligo (dT). For the PCR amplification of cDNA, an initial amplification step using $Z A P$-specific primers was performed with denaturation at $95^{\circ} \mathrm{C}$ for $10 \mathrm{~min}$, followed by 40 denaturation cycles at $95^{\circ} \mathrm{C}$ for $10 \mathrm{~s}$ and primer annealing/extension at $61^{\circ} \mathrm{C}$ for $30 \mathrm{~s}$. A final extension step was performed at $72^{\circ} \mathrm{C}$ for $5 \mathrm{~min}$ after the denaturation cycles were completed, after which the reaction mixtures were stored at $4^{\circ} \mathrm{C}$. Specific gene expression was quantified by using SuperReal PreMix SYBR Green (FP204-02, TIANGEN, Beijing, China) with an Applied Biosystems 7500 Fast Real-Time PCR system (Life Technologies). All gene expression levels were normalized to $\beta$-actin. The following amplification primers (Thermo Fisher Scientific, Waltham, MA) were used: ZAP sense (TCACGAACTCTCTGGACTGAA); ZAP antisense (ACTTTTGCATATCTCGGGCATAA); $\beta$-actin sense (GATCATTGCTCCTCCTGAGC); and $\beta$-actin antisense (ACTCCTGCTTGCTGATCCAC).

\section{Antibodies and western blot analyses}

Cells at $70 \%$ to $80 \%$ confluence were washed twice with ice-cold phosphate-buffered saline (PBS) and lysed on ice in Mammalian Protein Extraction Reagent (M-PER; Thermo Fisher Scientific) containing a complete protease inhibitor cocktail (Roche Applied Sciences, Penzberg, Germany). Fresh tissue samples were ground to powder in liquid nitrogen and then lysed with sodium dodecyl sulfate-polyacrylamide gel electrophoresis (SDS-PAGE) sample buffer. Equal amounts of protein samples (20 $\mu \mathrm{g}$ ) were separated on $10 \%$ SDS-PAGE and then transferred to polyvinylidene fluoride membranes (Immobilon P, Millipore, Billerica, MA). The membranes were blocked with $5 \%$ fat-free milk in tris-buffered saline containing $0.1 \%$ Tween-20 for $1 \mathrm{~h}$ at room temperature. The membranes were incubated first with primary antibody overnight at $4{ }^{\circ} \mathrm{C}$, and then with the appropriate horseradish peroxidase (HRP)-conjugated secondary antibodies. The proteins were visualized with a Chemi Doc XRS+ System (Bio-Rad Laboratories, Hercules, CA) using Immobilon Western Chemiluminescent HRP Substrate (Millipore). The antibodies used in this study are as follows: ZAP (PA5-31650; Thermo Fisher Scientific) and glyceraldehyde 3-phosphate dehydrogenase (GAPDH; AP0060; Bioworld Technology, Inc., St. Louis Park, MN).

\section{Clinical samples and clinical staging system}

This study assessed 147 paraffin-embedded HCC samples that were histopathologically and clinically diagnosed at the Sun Yat-sen University Cancer Center (State Key Laboratory of Oncology in South China, Guangzhou, China).

Clinical and clinical pathology classification and staging were determined in accordance with the criteria of the American Joint Committee on Cancer. Patient consent was obtained prior to the use of these clinical materials for research purposes. The patient consent form and the study protocol were approved by the Institutional Research Ethics Committee of our institution. The clinical data of the patients and samples are summarized in Table 1. The follow-up duration ranged from 3 months to 81 months; median

Table 1. Summary of patients' clinical and pathological characteristics and clinical samples. Abbreviations: AFP, alpha-fetoprotein; cm, centimeters; H, high; HBSAg, serum hepatitis B surface antigen; L, low; SD, standard deviation; ZAP, zinc finger antiviral protein

\begin{tabular}{lccc}
\hline & ZAP(L) & ZAP(H) & \\
& $\mathrm{n}=107$ & $\mathrm{n}=40$ & P-value \\
\hline HBSAg(+), \%, n/N & $76.6 \%, 82 / 107$ & $77.5 \%, 31 / 40$ & 0.9119 \\
Sex, n (M/W) & $83 / 24$ & $35 / 5$ & 0.1782 \\
Cirrhosis, \%, n/N & $56.1 \%, 60 / 107$ & $62.5 \%, 25 / 40$ & 0.4827 \\
AFP, mean \pm SD (U/ml) & $9369 \pm 2296$ & $5402 \pm 2166$ & 0.3213 \\
Size, mean \pm SD (cm) & $6.786 \pm 0.2854$ & $6.183 \pm 0.5617$ & 0.2991 \\
Grade, n (1/2/3/4) & $8 / 68 / 24 / 2$ & $8 / 22 / 7 / 3$ & 0.0611 \\
Stage, n (I/II/III/IV) & $17 / 32 / 49 / 9$ & $8 / 11 / 20 / 1$ & 0.5823 \\
T Classification, n (1/2/3/4) & $16 / 38 / 31 / 22$ & $11 / 13 / 11 / 5$ & 0.3051 \\
N Classification, n (0/1) & $104 / 4$ & $37 / 3$ & 0.3339 \\
M Classification, n (0/1) & $102 / 6$ & $40 / 0$ & 0.1280 \\
Vessel Invasion, \%, n/N & $52.3 \%, 56 / 107$ & $47.5 \%, 19 / 40$ & 0.6016 \\
Relapse, \%, n/N & $47.7 \%, 51 / 107$ & $47.5 \%, 19 / 40$ & 0.9859 \\
\hline & & &
\end{tabular}




\section{Cellular Physiology Cell Physiol Biochem 2018;49:1048-1059 \begin{tabular}{ll|l} 
and Biochemistry Published onlıne: 6 September, 2018 & (c) 2018 The Author(s). Published by S. Karger AG, Basel \\
ww.karger.com/cpb
\end{tabular}}

Ying et al.: Low ZAP Related with Progression and Poor Survival of HCC

follow-up duration was 30 months. For each patient, the presence of cirrhosis and the nature and size of the tumor were confirmed by final pathological examination. Serum AFP load (IU/mL) was determined and defined as negative when below the lower limit of detection $(20 \mathrm{U} / \mathrm{mL})$. Serum hepatitis B surface antigen (HBsAg) was detected with an enzyme-linked immunosorbent assay kit (KEHUANG Company, Shanghai, China).

Tissue microarray analysis of clinical samples

Tissue microarrays (TMAs) of the 147 clinical samples were provided by Dan Xie of the State Key Laboratory of Oncology in South China, Sun Yat-sen University Cancer Center. Eight matched HCC tissues and adjacent normal tissues were collected for each patient. Immunohistochemical (IHC) staining was performed on 5- $\mu \mathrm{m}$ sections of the TMAs to assess cytoplasmic expression of ZAP. TMA slides were scanned using the Aperio slide scanner and analyzed using Image Scope software (Aperio, Leica Biosystems Inc., Buffalo Grove, IL). The degree of immune staining of the formalin-fixed, paraffin-embedded sections was evaluated independently by two observers who were blind to the histopathological features and clinical data associated with each sample. The expression index scores given by the two independent investigators were averaged and were based on both the proportion of positively-stained tumor cells and the intensity of staining. The expression index score was derived as follows: score = proportion of cells with positive expression (0-10\%: 1; 10-25\%: 2; 25-50\%: 3; >50\%: 4) $\times$ mean intensity of staining (0-3). An optimal cut-off value was determined as follows: low expression of ZAP was defined as a ratio of (expression index score of tumor/expression index score of paired adjacent non-neoplastic tissue) $<1$ and normal or high expression of ZAP was defined as a ratio $\geq 1$.

Study approval

Use of the primary cancer tissue specimens was approved by the ethics review committee of Sun Yatsen University.

\section{Statistical analysis}

The primary endpoint of this study was the overall survival (OS). OS was defined as the duration from the date of each patient's random assignment to the date of death from any cause or the censoring of the patient at the date of the last follow-up. All statistical analyses were performed with the SPSS 20.0 statistical software package (IBM Corp., Armonk, NY). The relationship between ZAP expression and clinic pathological characteristics was analyzed with chi-square and Fisher's exact tests. Bivariate correlations between study variables were calculated as Spearman's rank correlation coefficients. Survival curves were plotted using the Kaplan-Meier method and compared with the log-rank test. Clinical and pathological characteristics typically used to predict prognosis in clinical practice were evaluated by using univariate and multivariate Cox regression analyses; we chose the forward method of Cox analysis. In all cases, a $P$-value of less than 0.05 was considered statistically significant.

\section{Results}

\section{ZAP expression is downregulated in liver cancer cell lines}

To evaluate the expression of ZAP in HCC, we first examined protein and mRNA expression in 11 HCC cell lines (HEP-3B, HUH-7, HUH-6, Sun-387, sun-449, SK-HEP-1, Sun182, Li-7, PLC, HEP-G2, and Bel-7402) and compared the observed expression to that of normal human hepatocytes (L-02 cells). The results showed that both ZAP mRNA and ZAP protein levels were significantly lower in the HCC cell lines than in normal hepatocytes (Fig. 1A, B). We also examined the ZAP mRNA and ZAP protein levels in primary HCC cultures and found that both were significantly lower in HCC cells than in normal hepatocytes (Fig. 2A, B).

\section{ZAP expression is down regulated in liver cancer tissues}

Molecular pathological methods were employed to detect ZAP expression in HCC tissues from 147 patients. For each patient, eight matched pairs of HCC tissue and adjacent normal tissue were subjected to TMA analysis with IHC for ZAP. In the samples of some patients, 
Fig. 1. Low expression of zinc finger antiviral protein (ZAP) protein and ZAP mRNA in hepatocellular carcinoma cell lines. (A and B). Expression of ZAP protein and ZAP mRNA in hepatocellular carcinoma cell lines (HEP-3B, HUH-7, HUH-6, Sun-387, Sun-449, SK-HEP-1, PLC, HEP-G2, and Bel-7402) and a normal liver cell line (L-02) was examined by western blot (A) and quantitative reverse transcription PCR (B), respectively. Expression levels were normalized against glyceraldehyde 3-phosphate dehydrogenase (GAPDH) (A) and $\beta$-actin (B). The error bars in $B$ represent the standard deviation of the mean (SD) calculated from three parallel experiments. $* \mathrm{P}<0.05$.

Fig. 2. Low expression of zinc finger antiviral protein (ZAP) protein and ZAP mRNA in primary cultured liver cancer cells. Expression of ZAP protein expression and ZAP mRNA

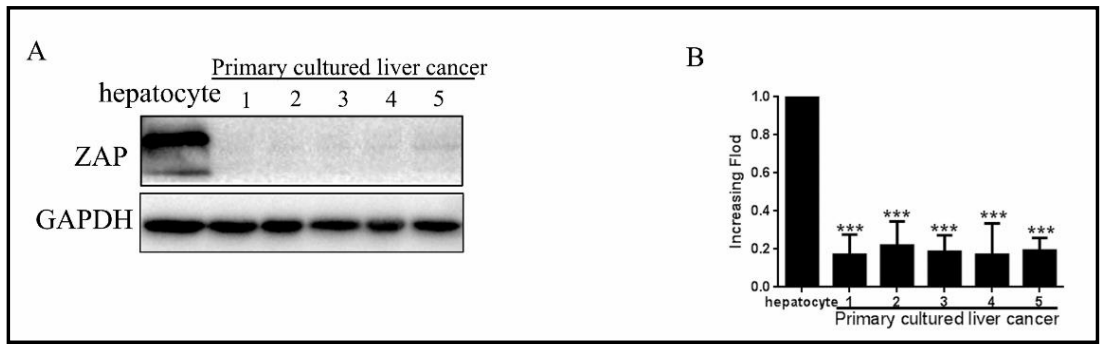

expression in normal hepatocytes (leftmost sample of A and B) and five primary cultures of liver cancer cells (remaining samples) was examined by western blot (A) and quantitative reverse transcription PCR (B), respectively. (A) glyceraldehyde 3-phosphate dehydrogenase (GAPDH) was the loading control. (B) Expression was normalized against GAPDH. The error bars in B represent the standard deviation of the mean (SD) calculated from three parallel experiments. ${ }^{*} \mathrm{P}<0.05$.

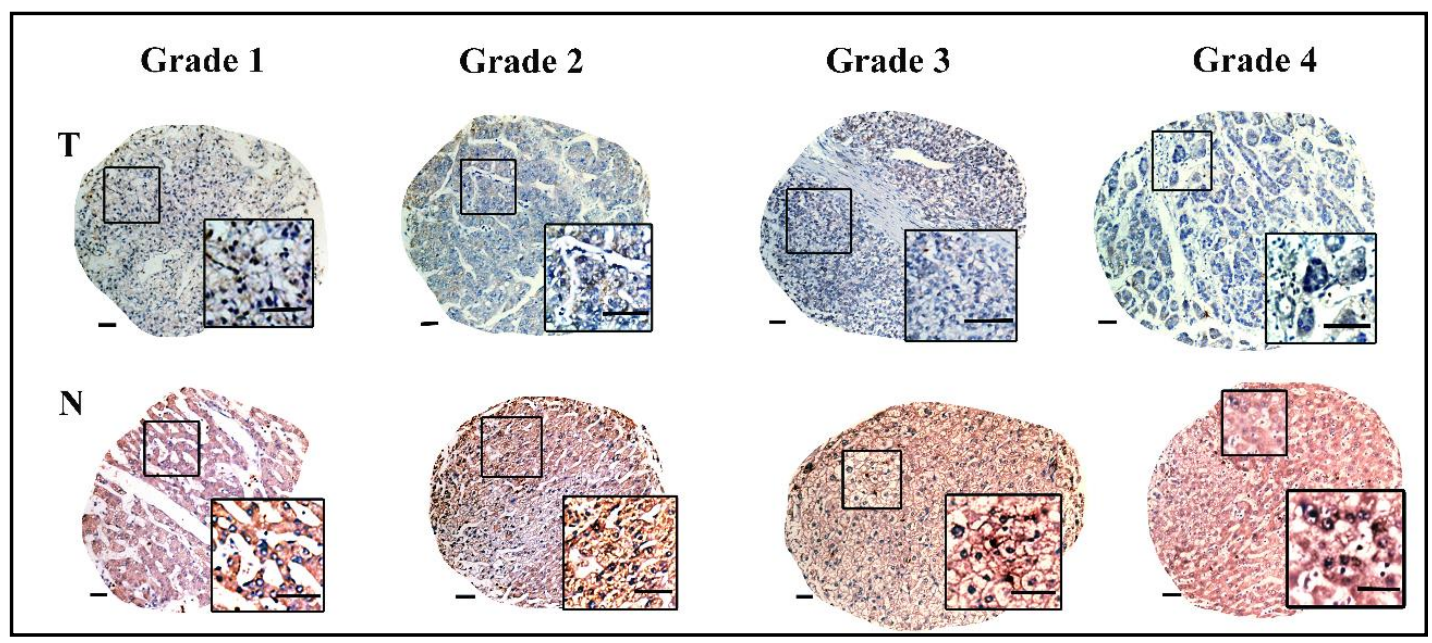

Fig. 3. Zinc finger antiviral protein (ZAP) protein expression in tumor samples. Top row: Representative images of ZAP protein expression in hepatocellular carcinoma tissues from patients with tumors of different grades: (A), grade 1; (B), grade 2; (C), grade 3; (D), grade 4. Bottom row: Immunohistochemical analysis of ZAP protein expression in four pairs of matched HCC tissues. The boxed area of each image is shown at higher magnification in the inset. (Scale bars, $50 \mu \mathrm{m}$ ). 
we observed dark brown staining in the adjacent normal tissues, suggesting high levels of ZAP expression. In contrast, ZAP protein expression was low in HCC tissues (Fig. 3). In addition, TMA analysis revealed that ZAP expression in HCC tissues was significantly lower than that of adjacent normal tissues for $77 \%$ of cases (107 samples). In contrast, only $23 \%$ of cases had normal or high expression of ZAP in HCC tissues.

\section{Association between ZAP expression and clinical features of patients with HCC}

To correlate the expression of ZAP with clinical characteristics of patients with HCC, the 147 patients who contributed samples to the TMAs were divided into two groups based on ZAP expression: normal or high (normal/ high) and low. We then compared clinical features of the groups: sex distribution, age, presence of cirrhosis or HBV infection, level of AFP, tumor size, tumor grade, clinical stage, $\mathrm{T}$ classification, $\mathrm{N}$ classification, $\mathrm{M}$ classification, presence of vessel invasion, and occurrence of relapse. No significant between-group differences were observed for any of these parameters (Table 1). Our analysis revealed that although patients with HCC and HBV infection were mainly male and had pre-existing hepatic cirrhosis, infection status was not associated with ZAP expression (Tables 2, 3). However, we discovered significant betweengroup differences in pathological grade among patients with both HCC and HBV infection $(P<0.05)$ : HBV-positive patients in the low expression group were under-represented for grade 1 (well differentiated) HCC $(45.5 \%$ of patients), but over-represented for grade 2 (moderately differentiated) HCC and grade $3 / 4$ (poorly or non-differentiated) HCC $(76.1 \%$ and $74.3 \%$ of patients, respectively), when compared to those in the high ZAP expression group (Table 4).

IHC analysis also revealed that clinical progression of HCC (i.e., an increase in clinical stage) was correlated with decreased expression of ZAP (Fig. 3). Among HBV-negative patients with HCC, tumor size was larger for patients in the low expression group than for those in the normal/high expression group $(6.944 \pm 0.5682 \mathrm{~cm}$ vs. $4.656 \pm 0.9567 \mathrm{~cm} ; P<$
Table 2. Correlation of the presence or absence of hepatitis B infection with clinical and pathological characteristics of patients and samples for patients in the low ZAP expression group. Abbreviations: AFP, alpha-fetoprotein; cm, centimeters; H, high; HBSAg, serum hepatitis $B$ surface antigen; L, low; SD, standard deviation; ZAP, zinc finger antiviral protein

\begin{tabular}{lccc}
\hline & HBsAg (-), ZAP(L) HBsAg (+), ZAP(L) \\
& $\mathrm{n}=25$ & $\mathrm{n}=82$ & \\
& & & \\
\hline Age, mean \pm SD, years & $50.04 \pm 2.287$ & $46.46 \pm 1.349$ & 0.1964 \\
Sex, n (M/W) & $12 / 13$ & $71 / 11$ & 0.0002 \\
Cirrhosis, \%, n/N & $28 \%, 7 / 25$ & $64.6 \%, 53 / 82$ & 0.0012 \\
AFP, mean \pm SD, (U/ml) & $2166 \pm 954.4$ & $11565 \pm 2944$ & 0.0832 \\
Size, mean \pm SD, (cm) & $6.944 \pm 0.5682$ & $6.738 \pm 0.3314$ & 0.7614 \\
Grade, n (1/2/3/4) & $3 / 17 / 5 / 0$ & $5 / 51 / 24 / 2$ & 0.5297 \\
Stage, n (I/II/III/IV) & $2 / 9 / 11 / 3$ & $15 / 23 / 37 / 6$ & 0.5378 \\
T Classification, n (0/1/2/3/4) & $4 / 10 / 4 / 7$ & $12 / 28 / 27 / 15$ & 0.3945 \\
N Classification, n (0/1/2/3) & $25 / 0 / 0 / 0$ & $78 / 4 / 0 / 0$ & 0.2604 \\
M Classification, n (0/1) & $23 / 2$ & $42 / 82$ & 0.6753 \\
Relapse, \%, n/N & $44 \%, 11 / 25$ & $48.8 \%, 40 / 82$ & 0.6753 \\
\hline & & & \\
\hline
\end{tabular}

Table 3. Correlation of the presence or absence of hepatitis B infection with clinical and pathological characteristics of patients and samples for patients in the high ZAP expression group. Abbreviations: AFP, alpha-fetoprotein; cm, centimeters; H, high; HBSAg, serum hepatitis B surface antigen; L, low; SD, standard deviation; ZAP, zinc finger antiviral protein

\begin{tabular}{lccc}
\hline & HBsAg (-), ZAP(H) HBsAg $(+)$, ZAP(H) \\
& $\mathrm{n}=9$ & $\mathrm{n}=31$ & \\
& & & \\
\hline Age, mean \pm SD, years & $52.44 \pm 3.749$ & $45.84 \pm 2.511$ & 0.2025 \\
Sex, n (M/W) & $6 / 3$ & $29 / 2$ & 0.0318 \\
Cirrhosis, \%, n/N & $33 \%, 3 / 9$ & $80.0 \%, 22 / 31$ & 0.0401 \\
AFP, mean \pm SD (U/ml) & $445.6 \pm 220.8$ & $6841 \pm 2749$ & 0.2220 \\
Size, mean \pm SD (cm) & $4.656 \pm 0.9567$ & $6.626 \pm 0.6551$ & 0.1452 \\
Grade, n (1/2/3/4) & $2 / 6 / 1 / 0$ & $6 / 16 / 6 / 3$ & 0.6920 \\
Stage, n (I/II/III/IV) & $4 / 3 / 2 / 0$ & $3 / 9 / 18 / 1$ & 0.0737 \\
T Classification, n (0/1/2/3/4) & $4 / 4 / 1 / 0$ & $7 / 9 / 10 / 5$ & 0.2301 \\
N Classification, n (0/1/2/3) & $8 / 1 / 0 / 0$ & $29 / 2 / 0 / 0$ & 0.6404 \\
M Classification, n (0/1) & $9 / 0$ & $16 / 31$ & 0.4182 \\
Relapse, \%, n/N & $55.5 \%, 5 / 9$ & $45.2 \%, 14 / 31$ & 0.5825
\end{tabular}


Table 4. Correlation of ZAP expression with clinic pathological characteristics of patients and samples among HBV-positive patients. Abbreviations: AFP, alpha-fetoprotein; $\mathrm{cm}$, centimeters; H, high; HBSAg, serum hepatitis B surface antigen; L, low; SD, standard deviation; ZAP, zinc finger antiviral protein

\begin{tabular}{lccc}
\hline & HBsAg $(+), \mathrm{ZAP}(\mathrm{L}) \mathrm{HBsAg}(+), \mathrm{ZAP}(\mathrm{H})$ & \\
& $\mathrm{n}=82$ & $\mathrm{n}=31$ & \\
& & & \\
\hline Age, mean \pm SD, years & $46.46 \pm 1.349$ & $45.84 \pm 2.511$ & 0.8162 \\
Sex, n (M/W) & $71 / 11$ & $29 / 2$ & 0.3007 \\
Cirrhosis, \%, n/N & $64.6 \%, 53 / 82$ & $80.0 \%, 22 / 31$ & 0.5249 \\
AFP, mean \pm SD (U/ml) & $11565 \pm 2944$ & $6841 \pm 2749$ & 0.3549 \\
Size, mean \pm SD (cm) & $6.738 \pm 0.3314$ & $6.626 \pm 0.6551$ & 0.8680 \\
Grade, n (1/2/3/4) & $5 / 51 / 24 / 2$ & $6 / 16 / 6 / 3$ & 0.0464 \\
Stage, n (I/II/III/IV) & $15 / 23 / 38 / 6$ & $3 / 9 / 18 / 1$ & 0.5149 \\
T Classification, n (1/2/3/4) & $12 / 28 / 27 / 15$ & $7 / 9 / 10 / 5$ & 0.7802 \\
N Classification, n (0/1) & $78 / 4$ & $29 / 2$ & 0.7393 \\
M Classification, n (0/1) & $78 / 4$ & $31 / 0$ & 0.2105 \\
Vessel Invasion, \%, n/N & $51.2 \%, 42 / 82$ & $51.6 \%, 16 / 31$ & 0.9702 \\
Relapse, \%, n/N & $48.8 \%, 40 / 82$ & $45.2 \%, 14 / 31$ & 0.7311 \\
\hline & & & \\
\hline
\end{tabular}

Table 5. Correlation of ZAP expression with clinical and pathological characteristics of patients and samples among HBV-negative patients. Abbreviations: AFP, alpha-fetoprotein; cm, centimeters; H, high; HBSAg, serum hepatitis B surface antigen; L, low; SD, standard deviation; ZAP, zinc finger antiviral protein

\begin{tabular}{lccc}
\hline & HBsAg $(-), \mathrm{ZAP}(\mathrm{L}) \mathrm{HBs}$ (-) $\mathrm{ZAP}(\mathrm{H})$ & \\
& $\mathrm{n}=25$ & $\mathrm{n}=9$ & \\
& & & \\
\hline Age, mean \pm SD, years & $50.04 \pm 2.287$ & $52.44 \pm 3.749$ & 0.5908 \\
Sex, n (M/W) & $12 / 13$ & $6 / 3$ & 0.3360 \\
Cirrhosis, \%, n/N & $28 \%, 7 / 25$ & $33 \%, 3 / 9$ & 0.7633 \\
AFP, mean \pm SD (U/ml) & $445.6 \pm 220.8$ & $2166 \pm 954.4$ & 0.2937 \\
Size, mean \pm SD (cm) & $6.944 \pm 0.5682$ & $4.656 \pm 0.9567$ & 0.0469 \\
Grade, n (1/2/3/4) & $3 / 17 / 5 / 0$ & $2 / 6 / 1 / 0$ & 0.6810 \\
Stage, n (I/II/III/IV) & $2 / 9 / 11 / 3$ & $5 / 2 / 2 / 0$ & 0.0227 \\
T Classification, n (1/2/3/4) & $4 / 10 / 4 / 7$ & $4 / 4 / 1 / 0$ & 0.1766 \\
N Classification, n (0/1) & $25 / 0$ & $8 / 1$ & 0.0907 \\
M Classification, n (0/1) & $23 / 2$ & $9 / 0$ & 0.3818 \\
Vessel Invasion, \%, n/N & $56 \%, 14 / 25$ & $33.3 \%, 3 / 9$ & 0.2435 \\
Relapse, \%, n/N & $44 \%, 11 / 25$ & $55.5 \%, 5 / 9$ & 0.5515 \\
\hline
\end{tabular}

0.05; Table 5). Patients in the low expression group also exhibited more advanced clinical stages than did those in the normal/high expression group $(P<0.05)$ : samples from 5 of 7 patients with stage I HCC (71.4\%) showed normal or high ZAP expression, but samples from 9 of 11 patients with stage II HCC $(81.8 \%), 11$ of 13 patients with stage III HCC $(84.6 \%)$, and 3 of 3 patients with stage IV HCC $(100 \%)$ had low ZAP expression (Table 5). Taken together, these results indicate that low expression of ZAP is related to the clinical progression of HCC for patients who are not infected with HBV.

\section{Association between ZAP expression and patient survival}

Survival analysis showed that the cumulative OS of HCC patients was negatively correlated with a deficiency of ZAP protein expression in tumor samples $(P<0.05$; Fig. 4B). Further analysis was performed after patients were stratified by tumor stage and $\mathrm{T}$ classification. The results indicate that ZAP expression was correlated with OS for patients with moderately to well-differentiated HCC (stage 1-2, log-rank test, $P<0.05$ ), but not for those with poorly differentiated HCC (stage 3-4, log-rank test, $P=0.102$; Fig. 4C, D). In addition, low expression of ZAP in samples from patients with T1 and T2 HCC was significantly correlated with a lower OS (Fig. 4E, log-rank test, $P<0.05$ ). Moreover, Cox regression analysis revealed that low ZAP expression was an independent predictor of poor prognosis (Table 6). However, no association between ZAP expression and T classification was noted for patients with T3 and T4 HCC (Fig. 4F, log-rank test, $P=0.209$ ). 
Fig. 4. Analysis of the association between zinc finger antiviral protein (ZAP) protein expression and overall survival (OS). A-F show the results of uni- or multivariate analyses of OS with Cox-regression analysis of ZAP protein expression in patients with hepatocellular carcinoma (HCC). All panels compare OS for patients in the low and high ZAP expression groups. For A-F, the $\mathrm{x}$-axis shows survival time (in months); the $\mathrm{y}$-axis shows cumulative survival. For A-E, the solid line represents survival of the high ZAP expression group and the dashed line represents that of the low ZAP expression group. For F, the solid line represents survival of the low ZAP expression group and the dashed line represents that of the high ZAP expression group. (A) A KaplanMeier curve with univariate analysis (logrank). (B) Multivariate analysis of OS for all patients. (C) Multivariate analysis of OS for patients with Grade 1 or 2 HCC. (D) Multivariate analysis of OS for patients with Grade 3 or 4 HCC. (E) Multivariate analysis of OS for patients with T1 or T2 grade tumors. (F) Multivariate analysis of OS for patients with $\mathrm{T} 3$ or $\mathrm{T} 4$ grade tumors.

\section{Discussion}

Our results clearly indicate that mRNA and protein expression of ZAP in both immortalized HCC cell lines and primary HCC cells is reduced significantly compared to expression in normal hepatocytes and commercial primary hepatocytes. A histopathological microarray assay also indicates that ZAP expression was markedly lower in HCC tissues than in adjacent normal tissues. These findings are consistent with those reported by Lin et al. [12]: low ZAP expression was observed in $69 \%$ of patients with HCC, $52 \%$ of those with colon cancer, and $61 \%$ of those with bladder cancer. Low expression of ZAP has been observed in multiple cancers, and our study confirms that ZAP expression is decreased in HCC. Thus, the function of the ZAP gene in the occurrence and development of cancers, especially HCC, needs to be further elucidated.
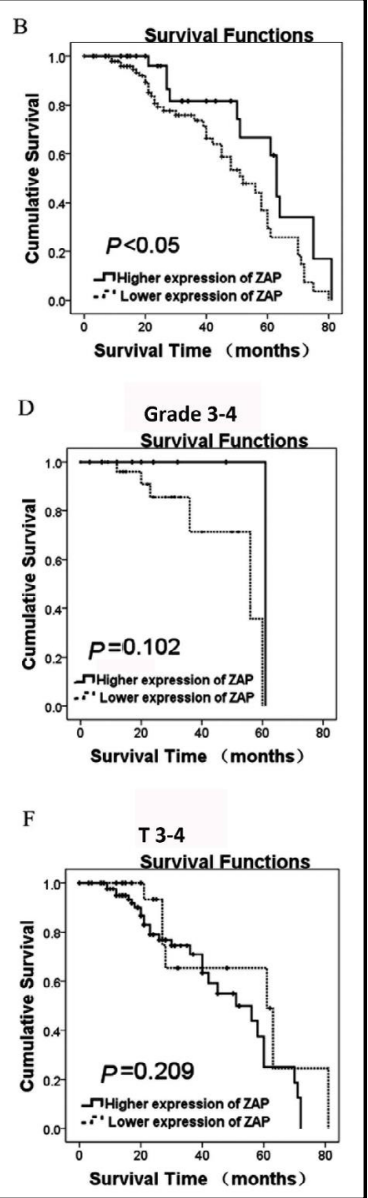

Table 6. Multivariate analyses* of prognostic parameters for patients with hepatocellular carcinoma. * Cox-regression analysis (overall survival was modeled). Abbreviations: HBSAg, Serum hepatitis B surface antigen; ZAP, zinc finger antiviral protein

\begin{tabular}{lcccc}
\hline & No. patients & P-value & Relative risk & $95.0 \%$ confidence interval \\
\hline ZAP & & & & \\
Low Expression of ZAP & 107 & 0.005 & 2.686 & $1.339-5.388$ \\
High Expression of ZAP & 40 & & & \\
HBSAg & 113 & 0.019 & 2.153 & $1.132-4.096$ \\
T Classification & & & & \\
T & & 0.007 & & \\
T1 & 27 & & & \\
T2 & 51 & 0.841 & 1.068 & $0.564-2.023$ \\
T3 & 42 & 0.014 & 2.675 & $1.216-5.886$ \\
T4 & 27 & 0.002 & 10.626 & $2.341-48.232$ \\
N Classification & & & & \\
N & & & & \\
N0 & 141 & $<0.001$ & 9.422 & $3.054-29.063$ \\
N1 & 7 & & & \\
Vessel Invasion & 75 & 0.003 & 4.286 & $1.617-11.356$ \\
\hline & & & & \\
\hline
\end{tabular}




\section{Cellular Physiology Cell Physiol Biochem 2018;49:1048-1059

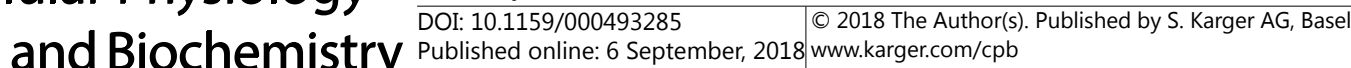

Ying et al.: Low ZAP Related with Progression and Poor Survival of HCC

IHC and analysis of clinical characteristics showed that low ZAP expression was found primarily in grade 2 and grade 3/4 tumors of patients infected with HBV; in HBV-negative patients with HCC, low ZAP expression was associated with tumor size and clinical stage. Our results indicate that decreased ZAP expression is associated with disease progression of HCC. Previous studies have demonstrated that the carcinogenesis of HCC is associated with the mutation or activation of numerous genes $[8,9,13]$. In the present study, HBV-positive patients with HCC often had hepatic cirrhosis and were mainly male, features consistent with the characteristics of patients with HBV infection [14]. The clinical parameters that were correlated with low ZAP expression differed between HCC patients who were HBVpositive and those who were HBV-negative. However, the specific mechanisms that give rise to these differences are still unclear and needed to be examined in detail.

In this study, we discovered that decreased ZAP expression was associated with a poorer prognosis and shorter survival of patients with HCC, particularly those with moderately- to well differentiated HCC (Grade 1-2) or stage T1 or T2 HCC. Multivariate Cox analysis also revealed that low expression of ZAP was an independent predictor of survival in HCC patients. These findings suggest that ZAP expression may be a prognostic indicator for patients with Grade $1 / 2$ or stage T1/2 HCC. In our study, multivariate Cox analysis indicated that HBV infection, $\mathrm{T}$ classification, $\mathrm{N}$ classification, and vessel invasion were independent predictors of poorer cumulative OS, all of which are consistent with previous reports [15-18]. Similarly, human metallopanstimulin-1 (MPS-1) is a multifunctional ribosomal S27/nuclear zinc finger protein that is highly expressed in actively proliferating cells and tumor tissues. It may be involved in the growth of aggressive breast cancer and the carcinogenesis of hepatoma [19, 20]. Fernandez-Pol et al. [21,22] demonstrated its key role in growth regulation in Archaea, eukaryotic cells, DNA repair, translation, and viral replication.

ZAP acts at the posttranscriptional level through regulating the stability of RNA, and interfering with translation of specific mRNAs and modulating the miRNA silencing pathway $[10,23-28]$. ZAP is an interferon-related gene with antiviral activity that may inhibit the replication of multiple viruses, including HBV, Moloney murine leukemia virus, human immunodeficiency virus 1, Ebola virus, Marburg virus, Sindbis virus, and Ross River virus $[23,24]$. ZAP may recognize and bind to the RNA of HBV and then degrade viral RNA, exerting an antiviral effect $[10,23,25,26]$. It is well known that HBV infection confers an increased risk of HCC, and that infection can cause HCC [2]. After HBV infection, chronic inflammation and altered expression of host genes are responsible for the pathogenesis of HCC $[27,28]$. ZAP functions in HBV-infected hepatocytes primarily during the acute phase, and we suspect that its activity may prevent progression into chronic infection and reduce the occurrence of HBV-related HCC [28]. In addition, ZAP also represses the expression of cellular RNAs through RNA decay. Todorova et al. [29] found that the antitumor effects of ZAP arise from its ability to bind cellular RNA to promote immune responses mediated by TRAIL and to regulate the pro inflammatory and pro apoptotic effects of TRAIL receptor 4. A recent study has reported that TRAIL may inhibit cancer growth and improve survival in mice [30]. Thus, we speculate that ZAP may regulate some signaling pathways to affect HCC carcinogenesis. As has been observed in HCC, studies have indicated that ZAP expression may prevent leukemia in mice by inhibiting the replication of MLV [25].

Just like MPS-1 protein, its increased level in patients is useful for early detection, prevention and treatment $[20,31]$. ZAP may thus be a useful marker to better understand the biological behavior of HCC and may become a new target for the development of an anticancer drug because of its ability to block the progression of HCC. A glycogen synthase kinase 3inhibitor has been tested as an antitumor drug in phase 2-3 clinical trials, and the results indicate that it can inhibit ZAP phosphorylation to exert anti-inflammatory and antitumor effects [32]. In addition, there is evidence that infection of cancer cells with an oncolytic virus may have an anti-tumor effect in cells with low ZAP expression [12]. 


\section{Cellular Physiology Cell Physiol Biochem 2018;49:1048-1059 \begin{tabular}{l|l|l} 
and Biochemistry & Dublished online: 6 September, 2018 & $\begin{array}{l}\text { C } 2018 \text { The Author(s). Published by S. Karger AG, Basel } \\
\text { www.karger.com/cpb }\end{array}$
\end{tabular}}

\section{Conclusion}

Our results indicate that low ZAP expression is common in HCC, is correlated with the progression of HCC and with a poorer prognosis for HCC patients, and plays an important role in HCC carcinogenesis. More clinical studies with larger sample sizes are needed to elucidate the correlation between HCC and ZAP expression. It will also be useful to identify ZAP as a tumor suppressor gene and to develop ZAP as a new target for the treatment of HCC.

\section{Abbreviations}

HCC (hepatocellular carcinoma); ZAP (zinc finger antiviral protein); TRAIL (tumor necrosis factor-related apoptosis inducing ligand); HBV (hepatitis B virus); AFP (alphafetoprotein).

\section{Acknowledgements}

This work was funded by the National Natural Science Foundation of China (Nos. 81570539, 81672701, and 81370535), the Science and Technology Planning Project of Guangdong Province, China (Nos. 20160909, 411308023039, and 2016A020215221), the Natural Science Foundation of Guangdong Province (No. 2017A030313620), the Research and Development Project of Applied Science and Technology of Guangdong Province, China (No. 2016B020237004), and the Guangzhou Science and Technology Project (No. 1561000155). The funding sources had no role in the study design, data collection, and analysis; decision to publish; or preparation of the manuscript.

We thank Dr. Dan Xie for providing tissue microarrays.

All data and materials are well documented.

There is no issue about ethics approval and consent to participate in the study. Use of the primary cancer tissue specimens was approved by the ethics review committee of Sun Yat-sen University.

All the authors have read and approved the paper and declare no potential conflicts of interest in the paper. If their paper is accepted, all the authors will observe the terms of the license to publish.

YL collected the tissue specimens and patient information. $\mathrm{CH}$ performed the statistical analyses. W-BZ drafted the manuscript. W-XX performed the western blotting. Z-YL participated in collecting patient information. YL edited the manuscript. JC performed the immunohistochemical analysis. J-KL conducted RNA extraction and real-time PCR. XZ participated in conceiving the study and guiding the editing of the manuscript. Z-LG supported this project. G-MY supervised the project. LP conceived the study, wrote the manuscript, and guided the editing of the manuscript. All authors read and approved the final manuscript.

\section{Disclosure Statement}

The authors declare that they have no competing interests.

\section{References}

\footnotetext{
1 McGuire S: World Cancer Report 2014. Geneva, Switzerland: World Health Organization, International Agency for Research on Cancer, WHO Press, 2015. Adv Nutr 2016;7:418-419.

-2 El-Serag HB: Epidemiology of viral hepatitis and hepatocellular carcinoma. Gastroenterology 2012;142:1264-1273.
} 


\section{Cellular Physiology Cell Physiol Biochem 2018;49:1048-1059 \begin{tabular}{ll|l} 
and Biochemistry Published online: 6 September, 2018 & (c) 2018 The Author(s). Published by S. Karger AG, Basel \\
ww.karger.com/cpb
\end{tabular}}

Ying et al.: Low ZAP Related with Progression and Poor Survival of HCC

3 Hanahan D, Weinberg RA: The hallmarks of cancer. Cell 2000;100:57-70.

4 Feitelson MA, Sun B, Satiroglu Tufan NL, Liu J, Pan J, Lian Z: Genetic mechanisms of hepatocarcinogenesis. Oncogene 2002;21:2593-2604.

-5 Lee JW, Soung YH, Kim SY, Lee HW, Park WS, Nam SW, Kim SH, Lee JY, Yoo NJ, Lee SH: PIK3CA gene is frequently mutated in breast carcinomas and hepatocellular carcinomas. Oncogene 2005;24:1477-1480.

6 Liston P, Fong WG, Korneluk RG: The inhibitors of apoptosis: there is more to life than Bcl2. Oncogene 2003;22:8568-8580.

-7 Suzuki K, Hayashi N, Yamada Y, Yoshihara H, Miyamoto Y, Ito Y, Ito T, Katayama K, Sasaki Y, Ito A, et al.: Expression of the c-met protooncogene in human hepatocellular carcinoma. Hepatology 1994;20:12311236.

8 Wang XW, Forrester K, Yeh H, Feitelson MA, Gu JR, Harris CC: Hepatitis B virus X protein inhibits p53 sequence-specific DNA binding, transcriptional activity, and association with transcription factor ERCC3 Proc Natl Acad Sci U S A 1994;91:2230-2234.

-9 Zender L, Spector MS, Xue W, Flemming P, Cordon-Cardo C, Silke J, Fan ST, Luk JM, Wigler M, Hannon GJ, $\mathrm{Mu}$ D, Lucito R, Powers S, Lowe SW: Identification and validation of oncogenes in liver cancer using an integrative oncogenomic approach. Cell 2006;125:1253-1267.

10 Zhu Y, Wang X, Goff SP, Gao G: Translational repression precedes and is required for ZAP-mediated mRNA decay. EMBO J 2012;31:4236-4246.

11 Todorova T, Bock FJ, Chang P: Poly(ADP-ribose) polymerase-13 and RNA regulation in immunity and cancer. Trends Mol Med 2015;21:373-384.

12 Lin Y, Zhang H, Liang J, Li K, Zhu W, Fu L, Wang F, Zheng X, Shi H, Wu S, Xiao X, Chen L, Tang L, Yan M, Yang X, Tan Y, Qiu P, Huang Y, Yin W, Su X, Hu H, Hu J, Yan G: Identification and characterization of alphavirus M1 as a selective oncolytic virus targeting ZAP-defective human cancers. Proc Natl Acad Sci U S A 2014;111:E4504-4512.

13 Nault JC, Zucman-Rossi J: Genetics of hepatobiliary carcinogenesis. Semin Liver Dis 2011;31:173-187. De Maria N, Manno M, Villa E: Sex hormones and liver cancer. Mol Cell Endocrinol 2002;193:59-63. Hwang S, Lee YJ, Kim KH, Ahn CS, Moon DB, Ha TY, Song GW, Jung DH, Lee SG: The Impact of Tumor Size on Long-Term Survival Outcomes After Resection of Solitary Hepatocellular Carcinoma: Single-Institution Experience with 2558 Patients. J Gastrointest Surg 2015;19:1281-1290.

-16 Rodriguez-Peralvarez M, Luong TV, Andreana L, Meyer T, Dhillon AP, Burroughs AK: A systematic review of microvascular invasion in hepatocellular carcinoma: diagnostic and prognostic variability. Ann Surg Oncol 2013;20:325-339.

17 Vauthey JN, Ribero D, Abdalla EK, Jonas S, Bharat A, Schumacher G, Lerut J, Chapman WC, Hemming AW, Neuhaus P: Outcomes of liver transplantation in 490 patients with hepatocellular carcinoma: validation of a uniform staging after surgical treatment. J Am Coll Surg 2007;204:1016-1027; discussion 1027-1018.

-18 Zhou L, Rui JA, Wang SB, Chen SG, Qu Q: Risk factors of microvascular invasion, portal vein tumor thrombosis and poor post-resectional survival in HBV-related hepatocellular carcinoma. Hepatogastroenterology 2014;61:1696-1703.

19 Ganger DR, Hamilton PD, Klos DJ, Jakate S, McChesney L, Fernandez-Pol JA: Differential expression of metallopanstimulin/S27 ribosomal protein in hepatic regeneration and neoplasia. Cancer Detect Prev 2001;25:231-236.

20 Fernandez-Pol JA, Hamilton PD, Klos DJ: Genomics, Proteomics and Cancer: Specific Ribosomal, Mitochondrial, and Tumor Reactive Proteins Can Be Used as Biomarkers for Early Detection of Breast Cancer in Serum. Cancer GenomProteom 2005;2:1-24.

21 Fernandez-Pol JA: Conservation of multifunctional ribosomal protein metallopanstimulin-1 (RPS27) through complex evolution demonstrates its key role in growth regulation in Archaea, eukaryotic cells, DNA repair, translation and viral replication. Cancer Genom Proteom 2011;8:105-126.

22 Sundblad AS, Ricci L, Xynos FP, Fernandez-Pol JA: Metallopanstimulin / S27 Ribosomal Antigen Expression in Stages I and II Breast Cancer: its Relationship with Clinicopathologic Factors. Cancer Genom Proteom 2005;2:53-60.

-23 Chen S, Xu Y, Zhang K, Wang X, Sun J, Gao G, Liu Y: Structure of N-terminal domain of ZAP indicates how a zinc-finger protein recognizes complex RNA. Nat Struct Mol Biol 2012;19:430-435.

24 Zhu Y, Gao G: ZAP-mediated mRNA degradation. RNA Biol 2008;5:65-67. 


\section{Cellular Physiology Cell Physiol Biochem 2018;49:1048-1059 $\begin{array}{lll}\text { DOI: 10.1159/000493285 } & \text { @ } 2018 \text { The Author(s). Published by S. Karger AG, Basel } \\ \text { and Biochemistry Published online: } 6 \text { September, } 2018 \text { www.karger.com/cpb }\end{array}$ \\ Ying et al.: Low ZAP Related with Progression and Poor Survival of HCC}

25 Gao G, Guo X, Goff SP: Inhibition of retroviral RNA production by ZAP, a CCCH-type zinc finger protein. Science 2002;297:1703-1706.

26 Mao R, Nie H, Cai D, Zhang J, Liu H, Yan R, Cuconati A, Block TM, Guo JT, Guo H: Inhibition of hepatitis B virus replication by the host zinc finger antiviral protein. PLoS Pathog 2013;9:e1003494.

27 Buendia MA, Neuveut C: Hepatocellular carcinoma. Cold Spring Harb Perspect Med 2015;5:a021444.

28 Cha MY, Kim CM, Park YM, Ryu WS: Hepatitis B virus X protein is essential for the activation of Wnt/betacatenin signaling in hepatoma cells. Hepatology 2004;39:1683-1693.

-29 Todorova T, Bock FJ, Chang P: PARP13 regulates cellular mRNA post-transcriptionally and functions as a pro-apoptotic factor by destabilizing TRAILR4 transcript. Nat Commun 2014;5:5362.

30 Hao C, Song JH, Hsi B, Lewis J, Song DK, Petruk KC, Tyrrell DL, Kneteman NM: TRAIL inhibits tumor growth but is nontoxic to human hepatocytes in chimeric mice. Cancer Res 2004;64:8502-8506.

-31 Fernandezpol JA: Increased serum level of RPMPS-1/S27 protein in patients with various types of cancer is useful for the early detection, prevention and therapy. Cancer Genomics Proteomics 2012;9:203-256.

-32 Cohen P, Goedert M: GSK3 inhibitors: development and therapeutic potential. Nat Rev Drug Discov 2004;3:479-487. 